\title{
El reto de la Vida
}

Independiente. La experiencia de la Fundación Síndrome de Down del País Vasco en la prestación de apoyos a Personas con Discapacidad Intelectual

\author{
The challenge for Independent Living. \\ Experience the Down Syndrome Foundation \\ of the Basque Country in providing support \\ for Intellectual Disability
}

\section{Palabras clave}

Discapacidad intelectual, programas

de apoyo, Filosofía de Vida

Independiente, Plan Individual de Emancipación.

\section{Keywords}

Intellectual disability, support programs, Independent Living Philosophy, Individual Plan of Emancipation.

\section{Introducción}

La Fundación Síndrome de Down del País Vasco (FSDPV), es una entidad privada, sin ánimo de lucro, que viene desarrollando su actividad desde el año I990; bajo la forma de Asociación Síndrome de Down de Bizkaia, primero, y como Fundación Síndrome de Down del País Vasco, desde el año I994.

La FSDPV tiene como misión mejorar la calidad de vida de las personas con discapacidad intelectual (DI), ofreciendo programas de apoyo de calidad adaptados a sus necesidades y a las de sus familias y que favorezcan su plena inclusión y participación social.

En este sentido, la evolución y crecimiento de la entidad ha estado marcado por la adecuación a las necesidades y reivindicaciones de las propias personas con DI a las que presta servicio. Así, se puede decir que a lo largo de estos ya más de 20 años, hemos ido realizado un camino
Laura Fernández Cordero <laurafernandez@downpv.org> Fundación Síndrome de Down del País Vasco

Para citar:

Fernández Cordero, L. (20I3): "El reto de la Vida Independiente. La experiencia de la Fundación Síndrome de Down del País Vasco en la prestación de apoyos a Personas con Discapacidad Intelectual". Revista Española de Discapacidad, I (2): I 59-I 65 .

$<$ http://dx.doi.org/I0.5569/23405IO4.OI.02.II> 
paralelo, durante el cual la entidad ha crecido a la par que lo han ido haciendo las personas a las cuales prestamos servicio.

La llegada de la edad adulta, nos trajo nuevos desafíos que, no sin pocas dificultades, hemos ido resolviendo. De entre todos estos retos, destaca el acceso al empleo ordinario y la constitución del grupo de autogestores ${ }^{\mathrm{T}}$. La salida al mercado laboral, acompañada de una mayor conciencia por parte del colectivo, del protagonismo que, respecto al control de su vida, le corresponde, marca un antes y un después en el diseño de programas y servicios durante esta etapa de su vida.

De esta manera, se activa también la reivindicación de un derecho fundamental: el acceso a una vida independiente en igualdad de condiciones al resto de ciudadanos/as.

Como respuesta a esta demanda, y en consonancia con las experiencias que en esta línea se venían desarrollando en distintos puntos del Estado, en el año 2004 la FSDPV, inició una línea de trabajo orientada a apoyar y fomentar el acceso de las personas con DI a una vida independiente basada en su elección personal.

Desde entonces han sido muchos los pasos que estas personas han dado y en los que se les ha ido acompañando: desde el paso por viviendas destinadas al aprendizaje y práctica de vida autónoma, hasta los actuales procesos de toma de decisión sobre cómo y dónde desean vivir.

En las siguientes páginas, se repasa la filosofía en la que se sustenta el proyecto, la situación actual del mismo y los retos que se plantean en un futuro.

I. El grupo de autogestores Entzun Gure Nahia, creado por la FSDPV, es desde el año 2005 una asociación independiente de la FSDPV, con entidad jurídica propia.

\section{Filosofía de partida}

\subsection{La Filosofía de Vida Independiente como referente}

Desde el Foro de Vida Independiente y Divertad $(\text { FVID })^{2}$ la Filosofía de Vida Independiente, se define como un pensamiento teórico-práctico en torno a la realidad humana, denominada "diversidad funcional" ${ }^{3}$ (discapacidad).

Desde esta filosofía se reclama el derecho de todas las personas con diversidad funcional a decidir sobre su propia existencia y participar activamente en la vida de su comunidad con los apoyos humanos necesarios. La herramienta fundamental para poder ejercitar estos derechos es la Asistencia Personal. Desde este movimiento se considera que sólo de esta manera las personas con Diversidad Funcional pueden tener el control de su propia vida.

Se rechaza la institucionalización como forma de vida, y el acceso a una vida autónoma e independiente se apunta como una cuestión de Derechos Humanos. Punto éste, ya asumido en la actualidad, en el artículo rg de la Convención de la ONU, sobre los Derechos de las Personas con Discapacidad.

La Filosofía de Vida Independiente se sustenta en 7 principios básicos:

- Derechos Humanos y civiles

- Autodeterminación

- Desinstitucionalización

- Desvinculación del modelo médico

- Empoderamiento

- Apoyo entre iguales

- Responsabilidad sobre la propia vida.

2. El Foro de Vida Independiente y Divertad es un grupo creado en año 200I, con intención de debatir y difundir la Filosofía de Vida Independiente, en internet. Se puede obtener mayor información sobre el grupo en $<w w w . f o r o v i d a i n d e p e n-$ diente.org $>$ y en <www.derechoshumanosya.org $>$.

3. Los miembros del FVID, se autodenominan personas con Diversidad Funcional, concepto por ellos acuñado como alternativa a discapacidad. 
El lema acuñado por el Movimiento de Vida Independiente refleja claramente el espíritu de esta filosofía: "Nada para nosotros sin nosotros".

El protagonismo y participación activa de la persona con diversidad funcional (discapacidad) a lo largo de todo el proceso adquiere en este paradigma máxima importancia. Han de ser ellos quienes decidan dónde, cómo y con quiénes desean vivir.

De esta forma, cada programa de vida independiente conforma una experiencia única, como únicas son cada una de las personas que forman parte de ellos. De acuerdo con los principios de autonomía y protagonismo de la persona con diversidad funcional (discapacidad), los programas han de ser diseñados con cada una de ellas y pensando en sus necesidades y deseos.

\subsection{La importancia de los apoyos}

Cuando se alude al término Vida Independiente, se hace desde el convencimiento de que toda persona con diversidad funcional (discapacidad), puede acceder a las mismas oportunidades y enfrentarse a las mismas elecciones en la vida diaria, de la misma manera que las personas sin discapacidad. Para ello, tiene que contar con los apoyos necesarios.

Cuando hablamos de prestación de apoyo, nos referimos a: "recursos y estrategias que promueven el bienestar de las personas y que tienen como resultado una mayor independencia y productividad personal, mayor participación en una sociedad interdependiente, mayor integración comunitaria y una mejor calidad de vida" (Thompson et al., 2002: 390)

Desde este paradigma, la detección de las capacidades y limitaciones de la persona se convierte en el medio para definir las adaptaciones y los tipos de apoyo que precise para llevar la vida más autónoma posible.

Ser independiente no significa hacerlo todo sin ayuda.

\section{EI proyecto ETXERATU}

\subsection{Los inicios}

Como ya se ha señalado, el Programa de Vida Independiente con apoyo ETXERATU, se inicia en el año 2004.

En estos primeros momentos, se pretendía ofrecer una primera experiencia de aprendizaje y entrenamiento a jóvenes adultos con diversidad/ discapacidad intelectual (DI) en las habilidades necesarias para vivir de manera independiente y autónoma, dentro de un piso compartido y con los apoyos necesarios en cada momento. Es muy importante señalar que la entrada en el programa no venía determinada por las necesidades de apoyo de cada persona con DI, por partir precisamente de esa Filosofía de Vida Independiente y entender ésta como un derecho humano básico e irrenunciable para todo ser humano.

En esta primera vivienda de estancias temporales, se estableció un sistema de participación rotatorio, según el cual cuatro personas con DI, apoyadas por profesionales de la FSDPV, pasan un período limitado a 3 meses y medio.

Durante este tiempo, los apoyos se dirigen específicamente a facilitar a la persona con DI la adquisición de las habilidades propias para llevar un hogar de manera autónoma, así como a favorecer un aumento de su autonomía y responsabilidad en otros aspectos complementarios como son la toma de decisiones, los desplazamientos, el control de horarios o las relaciones sociales con los compañeros y vecinos.

\subsection{Su evolución}

En el año 2007, con el Programa ETXERATU afianzado dentro del Servicio de Adultos, se realiza una valoración general del mismo. Como consecuencia de esa valoración, en el año 2008 se inicia la ampliación del Proyecto, reconvirtiéndole en un área de trabajo mucho más global, conformada por 3 programas, 
Figura 1. Proyecto ETXERATU

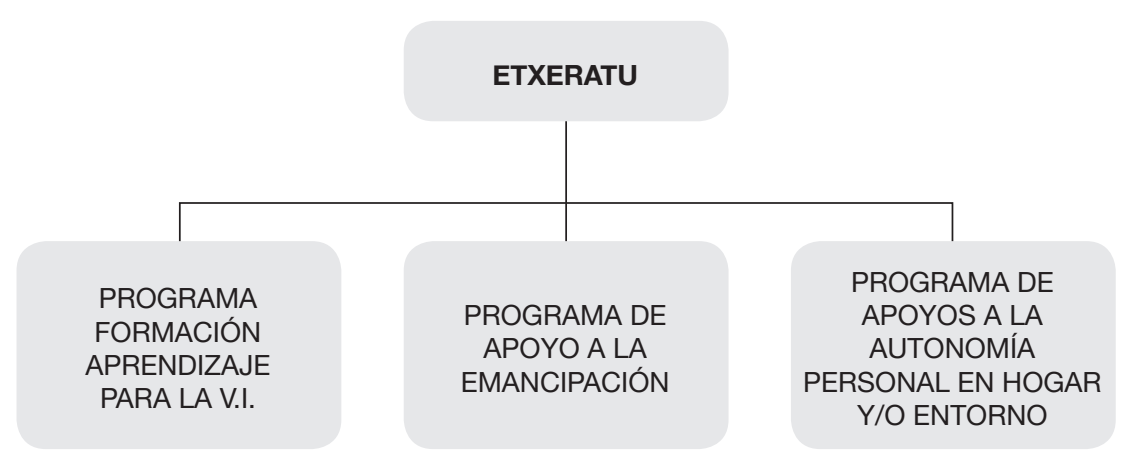

destinados a dar respuesta a las demandas de nuestros/as clientes:

\subsection{El programa de formación y aprendizaje para la vida independiente}

Se corresponde con el planteamiento inicial del programa. Se ofertan estancias de 3 o 6 meses en las Viviendas de Preparación para la Vida Autónoma (VPVA) de la entidad, a personas con DI mayores de I 8 años, que deseen iniciar un proceso de preparación para la vida independiente.

"El conocimiento ha de estar en la base de la elección" es la idea base alrededor de la cual se desarrolla este programa.

\subsection{Programa de apoyo a la emancipación}

El objetivo general de este programa es proporcionar a las personas con DI y sus familias todos los apoyos y recursos necesarios para que el proceso de transición hacia la vida independiente se desarrolle con éxito. Para ello se trabaja con cada uno/a de ellos/as y sus familias a través de los Planes Individuales de Emancipación.

El Plan Individual de Emancipación (PIE): es un plan individual consensuado entre la persona con DI, familia y FSDPV. Está destinado a concretar todas las acciones necesarias para que cada persona con DI interesada pueda llevar una vida independiente: ayudas municipales, inscripción en el servicio vasco de vivienda (ETXEBIDE), búsqueda de otras alternativas, etc.

Con el objetivo de cubrir las necesidades de adultos/as con DI que quieren o necesitan iniciar una vida independiente fuera del hogar familiar, pero que carecen de posibilidades de acceso a una vivienda propia, la FSDPV ha puesto en marcha una vivienda de estancias indefinidas (VEI) destinada a cubrir esta necesidad hasta que la persona pueda acceder a un recurso propio. En estos casos el PIE adquiere toda su importancia.

Una vez que la persona logra acceder a una vida emancipada, se pone en marcha la prestación de apoyos en ese nuevo escenario.

3.5. Programa de prestación de apoyos a la autonomía personal de personas con DI, en el hogar y entorno inmediato

El programa de prestación de apoyos a la autonomía personal pretende posibilitar y contribuir al desarrollo del proyecto de vida independiente y/o autónoma de cada persona con DI, en el entorno de hogar y entorno elegido por cada una de ellas y/o sus familias, a través de la prestación de un servicio individualizado y adaptado a sus necesidades y las de su familia. 
En el marco de este programa y atendiendo a las distintas demandas que las propias personas con DI y sus familias están reclamando, se responde no sólo a las necesidades derivadas del proceso de emancipación abordado en el programa anterior sino que también se atienden aquellas demandas formuladas en materia de promoción de autonomía personal. Por lo tanto, las principales necesidades pueden resumirse en los siguientes puntos:

- En materia de promoción de la autonomía personal: hay muchas personas con DI, que ya desde la etapa escolar precisan de ciertos apoyos concretos y específicos orientados a la adquisición de una mayor autonomía e independencia respecto a sus familiares, para poder desarrollar planes de vida ajustados a la etapa vital en la que se encuentran y que, con su prestación contribuyen a aumentar la inclusión y participación adecuada en la vida escolar y comunitaria.

- En apoyo al ejercicio de una vida independiente: Hay un gran número de personas con DI adultas, que demandan el derecho que como ciudadanos/as les asiste: el derecho a llevar una vida independiente definitiva (emancipación) en igualdad de condiciones al resto. Estas personas para tener éxito en la salida del hogar familiar han de contar con los apoyos necesarios en su nueva situación. Este tipo de apoyos se dirigen tanto al entorno de hogar como en el acceso a la vida comunitaria y el apoyo en gestiones de todo tipo: bancarias, en servicios sociales, visitas médicas, etc.

- En apoyo al mantenimiento de la persona con DI en el hogar: En tercer lugar, el aumento de la esperanza de vida de las personas con DI, ha traído consigo un incremento en el número de personas que están envejeciendo en sus hogares o en los de sus familias, sin contar en muchas ocasiones con los apoyos adecuados. En este caso el apoyo dentro del propio hogar familiar, como medio para lograr que cada uno de los miembros de la familia desarrolle su propia vida, se está revelando no sólo como una excelente fórmula para evitar la institucionalización de personas con DI de edad avanzada sino como medio igualmente válido para garantizar tiempos de descanso y dedicación a asuntos personales a los/as familiares que habitualmente se encargan de la supervisión y apoyo a la persona con DI; un "cuidar al cuidador/a" sin que nadie tenga que abandonar el hogar.

En base a estas necesidades, el programa se presta bajo 3 modalidades.

\section{Figura 2. Programa de Apoyos}

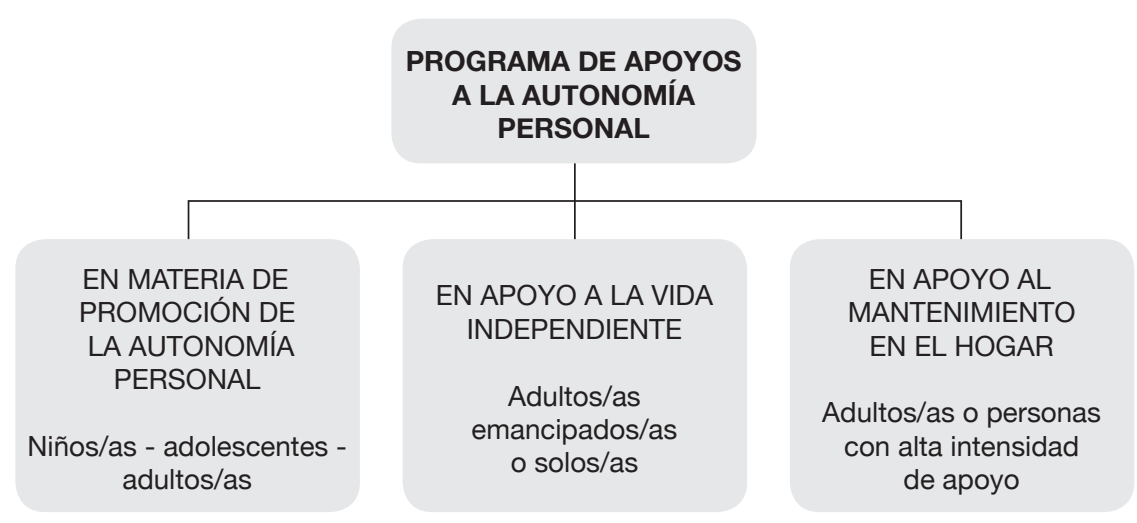


En todos los casos expuestos, la prestación de apoyos dentro del propio hogar y en el entorno es una solución adecuada y ajustada tanto para garantizar la permanencia en el hogar propio o familiar de las personas con DI más mayores, como para posibilitar la progresiva independencia y futura emancipación de las personas más jóvenes.

En base a estas necesidades, dentro del programa se prestan 2 tipos de apoyo:

\section{- Apoyo a personas con DI que viven} con sus padres y/o responsables legales. Desde cobertura de tiempos concretos, orientados principalmente a favorecer el desarrollo de su autonomía, hasta apoyos en períodos vacacionales o circunstancias extraordinarias. (situaciones de riesgo en la estructura familiar, por ejemplo).

- Apoyo a personas con DI que viven en su propio hogar. Apoyos destinados a garantizar la adecuada gestión del hogar y cobertura de las actividades básicas de la vida diaria de la persona con DI. Este tipo de apoyos se prestan lógicamente a personas adultas que bien han seguido un proceso de emancipación o por desaparición de la unidad familiar viven solas en su hogar.

\subsubsection{Metodología de trabajo del Programa de Apoyos en Hogar y Entorno inmediato}

Como se ha dicho con anterioridad, este es un programa que viene a dar respuesta a situaciones que, teniendo en común, la necesidad de apoyo bien para que la persona se mantenga en el hogar o bien para que acceda a una vida más autónoma o emancipada, requiere de soluciones totalmente individualizadas, que por lo tanto, van a ajustarse a la realidad de cada caso. Aún así, hay una metodología de trabajo que se desarrolla de la siguiente manera:

1. Demanda de apoyo: Es la persona con DI y/o su familia quien ha de formularla. Estas peticiones que, como ya se ha dicho, se realizan a lo largo de todo el año, hay que dirigirlas a la responsable del programa

2. Análisis de la petición: Una vez recibida la petición se analiza la viabilidad de su prestación.

Para ello se tiene en cuenta en primer lugar, si es una prestación destinada a cumplir con los objetivos de autonomía o emancipación a los que está destinado el programa. En segundo lugar, hay que realizar una primera valoración entre responsable del programa, persona con DI y familia para detallar los tiempos exactos y objetivos concretos de la prestación.

3. Selección de la persona de apoyo: Una vez analizada y aprobada la petición, se selecciona a la/s persona/s adecuada/s para prestar ese apoyo. Esa selección la realiza la persona responsable del programa y en el caso de personal ya conocido por el/ la usuario/a y su familia, contando con su participación y aprobación.

4. Reunión de planificación y seguimiento con persona de apoyo, familia y persona con DI: Una vez seleccionada la persona/a que va a cubrir el apoyo se inicia la ronda de reuniones necesarias para determinar las funciones que va a tener cada una de las partes: persona de apoyo, persona con DI, familia, etc. Esta parte es de importancia vital para el éxito posterior de la prestación, tanto en caso de apoyos en situación de emancipación o en casos que el hogar está compartido con algún miembro de la familia. Una vez iniciado el apoyo se establecen una serie de reuniones de seguimiento, para asegurar el buen funcionamiento del mismo. Es de gran importancia señalar que en el caso de las personas emancipadas su participación en la organización de la jornada laboral de la persona de apoyo (horas a cubrir; funciones a desempeñar; vacaciones, etc.) es total, garantizando así su control sobre la prestación de apoyo recibida.

5. Evaluación continua del apoyo: Igual de importante que hacer un seguimiento del 
buen funcionamiento del programa es medir los resultados obtenidos, de cara a poder establecer los cambios o modificaciones pertinentes que permitan avanzar en su prestación: cambiando el tipo de apoyo o reduciéndolo. Esta evaluación se realiza entre todas las partes implicadas: usuario/a, familia y persona de apoyo, con responsable.

\section{En conclusión}

En un momento como el actual, en el que tanto la más reciente legislación autonómica y nacional como la internacional, apuesta por fórmulas basadas en la prestación de apoyos como clave para respetar el derecho que cada persona con discapacidad tiene a decidir sobre dónde, cómo y con quién vivir, se hace necesario abordar este reto a través de la configuración de programas y servicios destinados a dicha causa.

Desde la FSDPV, entendemos que para que realmente un proyecto pueda ser considerado como de apoyo a la vida independiente ha de ser individualizado, ya que cada persona es única y únicas son las soluciones o apoyos que precise; e integral; es decir, ha de dar respuesta a todas las necesidades de la persona, no sólo a aquellas más básicas o de tipo doméstico, sino también a las que están orientadas a favorecer una igualdad de oportunidades en el acceso a todos los recursos y equipamientos públicos. Además se realiza una apuesta firme también por seguir una línea de fomento de la autonomía personal ya desde los primeros años de vida de la persona con DI, como principio que ha de regir toda intervención, ya que sólo educando en autonomía podremos contribuir a que los niños/ as y jóvenes con DI de hoy, tengan las máximas posibilidades de desarrollar proyectos de vida independiente en un futuro.

En este sentido, es fundamental que las instituciones públicas competentes en la materia, incorporen estos programas a la cartera de servicios y prestaciones, ofertados a personas con DI. Sólo con su apoyo se podrá continuar desarrollando actuaciones que, como ésta, pretenden hacer de los deseos, gustos e intereses de las personas con DI, los únicos elementos que marquen su participación activa en la vida comunitaria.

Este es nuestro reto y obligación, para los próximos años.

Referencias bibliográficas

ONU (2006): Convención sobre los Derechos de las Personas con Discapacidad y Protocolo Facultativo. Nueva York.

THOMPSON, J.R. et al. (2002): "Responding to the opportunities and challenges of the emerging supports paradigm: A suggested approach to planning, assessment and evaluation”. Mental Retardation, 40(5): 390. 